\title{
Modeling And Characterization of Carrier Mobility For Truncated Conical Quantum Dot Infrared Photodetectors
}

Yasser M. El-Batawy ( $\nabla$ ymbatawy@gmail.com )

Cairo University - Faculty of Engineering https://orcid.org/0000-0002-2960-8808

Marwa Feraig

Nile University School of Engineering and Applied Sciences

\section{Research Article}

Keywords: Quantum dot, QDIP (Quantum Dot Infrared Photodetector), Mobility, Photodetectors, Boltzmann Transport Equation

Posted Date: May 13th, 2021

DOI: https://doi.org/10.21203/rs.3.rs-446399/v1

License: (c) (i) This work is licensed under a Creative Commons Attribution 4.0 International License. Read Full License

Version of Record: A version of this preprint was published at Optical and Quantum Electronics on November 13th, 2021. See the published version at https://doi.org/10.1007/s11082-021-03346-4. 


\title{
Modeling and Characterization of Carrier Mobility for Truncated Conical Quantum Dot Infrared Photodetectors
}

\author{
Yasser M. El-Batawy ${ }^{(1),(2)}$ and Marwa Feraig ${ }^{(2)}$ \\ (1) Engineering Mathematics and Physics Department, Faculty of Engineering, Cairo University, Giza, Egypt, 12211 \\ ${ }^{(2)}$ Nanoelectronics Integrated Systems Center, Nile University, Giza, Egypt, 12588
}

\begin{abstract}
In the present paper, a full theoretical model for calculating the carrier mobility coming as a result of the existence of a truncated conical quantum dots of n-type quantum dot infrared photodetectors (QDIPs) is developed. This model is built on solving Boltzmann's transport equation that is a complex integro-differential equation describing the carrier transport. The timedomain finite-difference method is used in this numerical solution. The influences of dimensions and density of the QDs for this structure on the carrier mobility are studied. Eventually, the calculated mobility for truncated conical InAs/GaAs QDIP is contrasted to other conical, spherical, and hemispherical QD structures. The model put forward is a generic model that is applicable to various structures of truncated conical QDs devices.
\end{abstract}

Keywords: Quantum dot, QDIP (Quantum Dot Infrared Photodetector), Mobility, Photodetectors, Boltzmann Transport Equation.

\section{Introduction}

Quantum computing and quantum cryptography together warranted the escalating interest in quantum dot technologies [1]. This interest is in turn motivated by the inherent characteristics of the various quantum (well, wire, and dot) to tune the optical and electronic characteristics of various semiconductors making them more suitable for specific applications including thermal imaging, remote sensing, photovoltaics, and infrared photodetectors [2]-[3]. However, quantum dot infrared photodetectors (QDIPs) have particularly gained a significant momentum among modern and well-established infrared (IR) photodetectors including quantum well infrared photodetectors (QWIPs) and mercury-cadmium-telluride photodetectors (HgCdTe) since they have the following potential advantages, they: Retain high 3D carrier confinement of electron wave function, Show high normal photon absorption, Possess low dark current capacity; a major source of detection noise, Operate at high temperatures, Have extended photoexcitation lifetime and Exhibit phonon bottleneck effect [2]-[7] .

The multiple structural merits abovementioned have approbated extensive experimental and theoretical research on the design of QDIPs. Many theoretical and experimental works have conducted the enhancement of the design of these photodetectors by modifying the quantum dot 
parameters including shape, size, restrain and chemical composition [8]-[13]. Carrier mobility of the QD structure is considered as an important factor influencing major detector's parametric functions such as dark current, drift velocity, and electrical conductivity. Some theoretical models, however, did include carrier mobility in their designs yet with approximated models that did not prove efficient in real-world devices [14]. Building on our previous theoretical model of characterization of QDIPs with different structures of the quantum dots including conical, spherical and hemispherical QDIPs [15]-[16], the current work presents a complete theoretical modeling of the carrier mobility for truncated conical quantum dot structures that can be considered the most appropriate quantum dot shaped for describing the self-assembled QD structures. The presented model is based on solving the semi-classical Boltzmann transport equation (BTE), where the numerical solution of BTE is based on the finite difference time domain method. Finally, the carrier mobility of the truncated quantum dot is compared to the structures with conical, spherical, and hemispherical QDs. The proposed carrier mobility calculation in the present study is uniformly applicable across various QDIP-based semiconductors regardless of their chemical composition, quantum dot density, shape or size, or the number of quantum dot layers used. The truncated conical quantum dot is the most appropriate structure that describes the self-assembled quantum dot structures, where the conical QD most probably is not the fabricated shape. This paper is structured as follows, in section 2, a description of the structure of the truncated QDIP is presented, and a complete theoretical model to calculate the electron mobility is developed. In section 3, the impacts of the dimensions, aspect ratio and quantum density over the carrier mobility are investigated. Also, the calculated electron mobility is compared to those of other QD structures. Finally, a conclusion is presented in section 4.

\section{Electron Mobility for Truncated Conical QD Structure}

A schema of the self-assembled truncated conical quantum dots infrared photodetector (QDIP) is depicted in Figure 1 (a), where it contains of an optically active region that mainly consists of barrier material with distributed layers of self-assembled quantum dots deposited over a very thin wetting layer. This active region is sandwiched between top and bottom contacts. The basic cell of a truncated conical QD is shown in Figure 1 (b) with radius $r_{b}$ and height of $h_{b}$ where it contains a QD with of a bottom base of radius $r_{d}$, a upper base of radius $r_{0}$ and a height of $h_{d}$. 


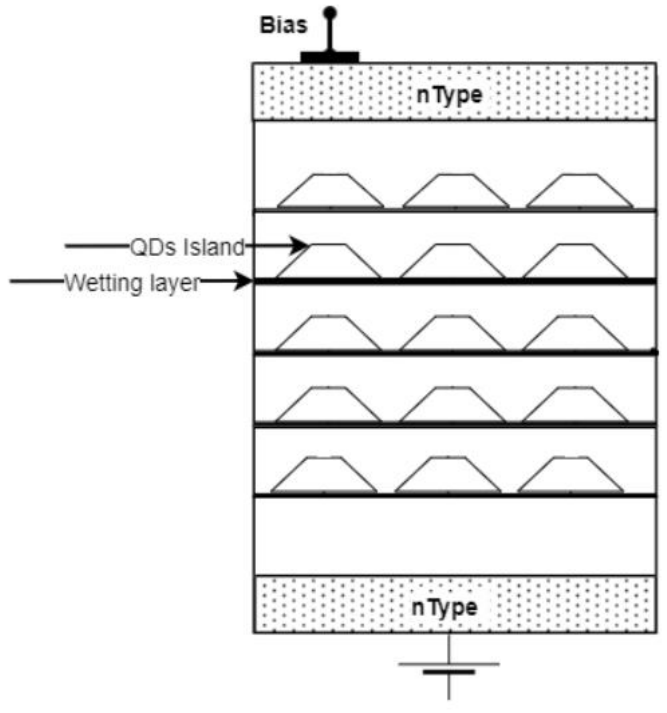

(a)

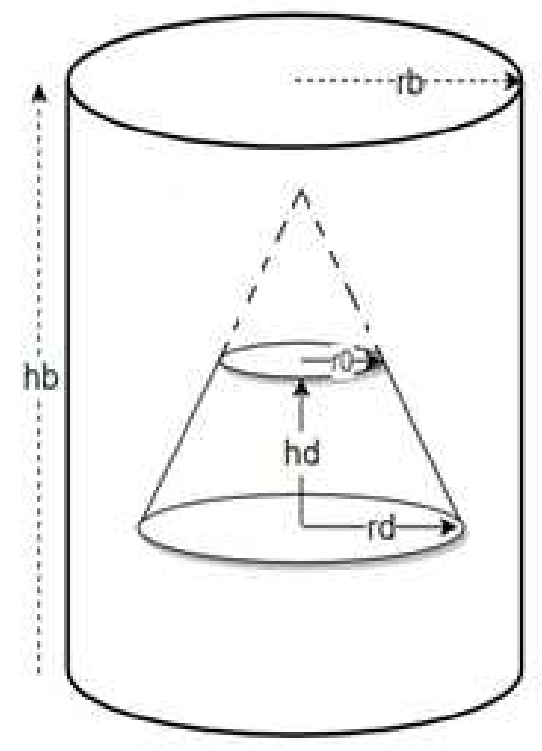

(b)

Figure 1: (a) Truncated conical QDIP structure (b) Basic cell of truncated conical QD structure

In the presented model of the carrier mobility, some reasonable assumptions are considered; (1) the quantum dots shape are considered to be truncated conical with insignificant variability in dimensions, (2) the influence of the very thin wetting layer underneath QDs is ignored, (3) the coupling between QDs can be ignored, since the QD size is relatively much smaller than the distance between them and the active region is thought of as a unitary medium where the QDs scatter carriers. To develop a complete theoretical method for computing the carrier mobility in QDIP, the Boltzmann transport equation will be solved to obtain the average total scattering electrons per unit time that affects the carrier mobility in the presence of the truncated conical QDs.

In the current study, the electron mobility of the truncated quantum dot is calculated by solving numerically the semi-classical Boltzmann transport equation (BTE). The procedure of this modeling is based on calculating the distribution function of the electrons, $f(\vec{r}, \vec{p}, t)[17]$. It is obvious that the distribution function must satisfy the continuity equation in the absence of collisions in a six-dimensional phase space, and it expresses the probability of finding electron in the state at position $\vec{r}$ with momenta $\vec{p}$ at time $t$. This distribution function is not dependent on $\vec{r}$, as the active region is thought of as a unitary homogeneous medium where QDs scatter carriers. Then, the change of the rate of $f_{p}$ associated with scattering is Formulated as 


$$
\left(\frac{\partial f_{p}}{\partial t}\right)_{\text {scattering }}=\frac{\partial f_{p}}{\partial t}+\frac{d \vec{p}}{d t} \cdot \nabla_{\vec{p}} f_{p}
$$

where $\frac{d \vec{p}}{d t}$ is the rate of change of the wave vector, which is just proportional to the force owing to the electric field $E$. The field term in BTE, $\frac{d \vec{p}}{d t} \cdot \nabla_{\vec{p}} f_{p}=\frac{q \vec{E}}{\hbar} . \nabla_{\vec{p}} f_{p}$, and the scattering term in BTE can be formulated as

$$
\left(\frac{\partial f_{p}}{\partial t}\right)_{\text {scat }}=\iiint\left[S_{p^{\prime}, p}\left(1-f_{p}\right) f_{p^{\prime}}-S_{p, p^{\prime}}\left(1-f_{p^{\prime}}\right) f_{p}\right] d^{3} p^{\prime} \frac{\Omega}{(2 \pi)^{3}},
$$

where $f_{p^{\prime}}$ and $f_{p}$ are the probabilities of finding the electron in energy levels $\varepsilon_{p^{\prime}}$ and $\varepsilon_{p}$, respectively. In the above equation, $S_{p^{\prime}, p}$ and $S_{p, p^{\prime}}$ are the transition rates from $p^{\prime}$ to $p$ and vice versa, respectively, and they can be calculated as

$$
S_{p^{\prime}, p}=S_{p, p^{\prime}}=\frac{2 \pi}{\hbar}\left|V_{p^{\prime}, p}\right|^{2} \delta\left(\varepsilon_{p^{\prime}}-\varepsilon_{p}\right)
$$

where $V_{p^{\prime}, p}$ is the matrix element of the perturbation $V(r)$ between the unperturbed and it is calculated according

$$
V_{p^{\prime}, p}=\frac{1}{\Omega} \tilde{V}\left(\overrightarrow{p^{\prime}}-\vec{p}\right),
$$

where $\tilde{V}\left(\overrightarrow{p^{\prime}}-\vec{p}\right)$ is the potential three-dimensional Fourier transform and $\Omega$ is the sample volume. The matrix elements are calculated for the truncated quantum dot structure, where it has upper and lower base radii of $r_{b}$ and $r_{o}$, respectively, and a height of $z_{o}$ while its vertex is within the zdirection. The external edges equations will be:

- $z$ : from 0 to $h_{d}$

- $y$ : from $y_{1}=-\sqrt{(z+l) \tan \theta_{0}}$ to $y_{2}=\sqrt{(z+l) \tan \theta_{0}}$ where $\theta_{0}=\tan ^{-1}\left(\frac{r_{o}}{l}\right)$

- $x$ : from $x_{1}=-\sqrt{(z+l)^{2} \tan \theta_{0}{ }^{2}-y^{2}}$ to $x_{2}=\sqrt{(z+l)^{2} \tan \theta_{0}{ }^{2}-y^{2}}$

Then, the matrix element perturbation owing to QDs with conduction band offset between the barrier and quantum dot $V_{b}$ is written as

$$
V_{f i}=-\frac{V_{b}}{\Omega} \int_{x=x_{1},}^{x=x_{2},} \quad \iint_{y=y_{1}, \quad z=0}^{y=y_{2}, \quad z=h_{d}} \exp \left(i\left(\vec{p}^{\prime}-\vec{p}\right) \cdot \vec{r}\right) d^{3} r
$$

where $\vec{p}$ and $\vec{p}^{\prime}$ are the wave vector that can expressed as $\vec{p}=p\left(\sin \theta_{p} \cos \varphi_{p} \hat{x}+\right.$ $\left.\sin \theta_{p} \sin \varphi_{p} \hat{y}+\cos \theta_{p} \hat{z}\right) \quad$ and $\quad \vec{p}^{\prime}=p^{\prime}\left(\sin \theta_{p^{\prime}} \cos \varphi_{p^{\prime}} \hat{x}+\sin \theta_{p^{\prime}} \sin \varphi_{p^{\prime}} \hat{y}+\cos \theta_{p^{\prime}} \hat{z}\right)$. Then, 


$$
\begin{aligned}
\left|\tilde{V}\left(\vec{p}^{\prime}-\vec{p}\right)\right|^{2}= & {\left[v_{b} \iint_{y=y_{1}, z=0}^{y=y_{2}, \quad z=h_{d}} \frac{2 \sin \left(p\left(\sin \theta_{p} \cos \varphi_{p}-\sin \theta_{p^{\prime}} \cos \varphi_{p^{\prime}}\right) x_{2}\right)}{p\left(\sin \theta_{p} \cos \varphi_{p}-\sin \theta_{k^{\prime}} \cos \varphi_{p^{\prime}}\right)}\right.} \\
& \left.\cdot \cos \left(p\left(\sin \theta_{p} \cos \varphi_{p}-\sin \theta_{p^{\prime}} \cos \varphi_{p^{\prime}}\right) y+\left(\cos \theta_{p}-\cos \theta_{p^{\prime}}\right) z\right)\right]^{2} \\
& +\left[v_{b} \iint_{y=-(z+l) \tan \theta_{o}, z=0}^{y=(z+l) \tan \theta_{o}, z=h_{d}} \frac{2 \sin \left(p\left(\sin \theta_{p} \cos \varphi_{p}-\sin \theta_{p^{\prime}} \cos \varphi_{p^{\prime}}\right) x_{2}\right)}{p\left(\sin \theta_{p} \cos \varphi_{p}-\sin \theta_{p^{\prime}} \cos \varphi_{p^{\prime}}\right)}\right. \\
& \left.\cdot \sin \left(p\left(\sin \theta_{p} \cos \varphi_{p}-\sin \theta_{p^{\prime}} \cos \varphi_{p^{\prime}}\right) y+\left(\cos \theta_{p}-\cos \theta_{p^{\prime}}\right) z\right)\right]^{2}
\end{aligned}
$$

In concordance with Matthiessen's rule, the average total scattering electrons per unit time depends on the scattering times by a number of microscopic scattering mechanisms and it can be expressed as

$$
\frac{1}{\tau}=\frac{1}{\tau_{\text {lattice }}}+\frac{1}{\tau_{\text {optical phonons }}}+\frac{1}{\tau_{\text {imputities }}}+\frac{1}{\tau_{\text {defects }}},
$$

where $\tau_{\text {lattice }}, \tau_{\text {optical phonons }}, \tau_{\text {impurities }}$, and $\tau_{\text {defects }}$ which are the mean free time associating with the scattering from lattice vibrations, optical phonons, impurities, and lattice defects such as quantum dots, respectively. In quantum dot devices, the most crucial factor in determining the carrier mobility in the active region is the scattering caused by the quantum dots [16], as the scattering from lattice vibrations and optical phonons have no significant impact on the mobility at low temperatures. Furthermore, due to the difference in sizes between the impurities and the quantized structures the scattering from the impurities is very small compared to the scattering from quantum dots. Then the scattering rate and the mobility of electrons in QPIDs is mainly influenced by QDs scattering mechanism that follows the Fermi's golden rule:

$$
\left(\frac{\partial f_{p}}{\partial t}\right)_{\text {scat }}=\frac{n_{Q D}}{(2 \pi)^{2} \hbar} \iiint\left(f_{p^{\prime}}-f_{p}\right)\left|\tilde{V}\left(\overrightarrow{p^{\prime}}-\vec{p}\right)\right|^{2} \delta\left(\varepsilon_{p^{\prime}}-\varepsilon_{p}\right) d^{3} p^{\prime},
$$

where $n_{Q D}$ is the QDs density. In the presented model, BTE equation is worked out numerically using the time-domain finite-difference method as the truncated conical QDs lack the spherical symmetry. As the truncated conical quantum dots have azimuthal symmetry and $\vec{E}$ does not depend on the wave vector $\vec{p}$, then

$$
\vec{E} . \nabla_{\vec{p}} f_{p}=\frac{1}{p^{2}} \frac{\partial}{\partial p}\left(p^{2} f_{p} E \cos \theta_{p}\right)-\frac{1}{p \sin \theta_{p}} \frac{\partial}{\partial \theta_{p}}\left(\sin ^{2}\left(\theta_{p}\right) f_{p} E\right) .
$$

Using the finite difference method, and the BTE can be represented as

$$
\begin{array}{r}
\frac{\partial f(p)}{\partial t}+\frac{q}{\hbar}\left(\frac{1}{p^{2}} \frac{\partial}{\partial p}\left(p^{2} f_{p} E \cos \theta_{p}\right)-\frac{1}{p \sin \theta_{p}} \frac{\partial}{\partial \theta_{p}}\left(\sin ^{2}\left(\theta_{p}\right) f_{p} E\right)\right) \\
=\frac{n_{\mathrm{QD}}}{(2 \pi)^{2} \hbar} \iiint\left(f_{p^{\prime}}-f_{p}\right)\left|\tilde{V}\left(\vec{p}^{\prime}-\vec{p}\right)\right|^{2} \delta\left(\varepsilon_{p^{\prime}}-\varepsilon_{p}\right) d^{3} p^{\prime}
\end{array}
$$


First, in the absence of the electric field, an initial distribution function is assumed, then the perturbation matrix element is computed for each point of $\left(p, \theta_{p}, p^{\prime}, \theta_{p^{\prime}}, \varphi_{p^{\prime}}\right)$. The collision term for each point of $\left(p, \theta_{p}\right)$ is calculated using the distribution function and the matrix element.at the same time, The field term for each point of $\left(p, \theta_{p}\right)$ is computed for a certain value of electric field using the distribution function. In order to subsequently solve the Boltzmann transport equation, the two terms of field and collision are used to define the new distribution function value by recruiting the time-domain-finite-difference methodology. Using the trapezoidal rule, the above integration is done numerically and using time-domain finite-difference method. Equation (10) can be rewritten as

$$
\begin{aligned}
& f(t+\Delta t)=f(t) \\
& +\Delta t .\left(\begin{array}{c}
-\frac{q E}{\hbar}\left(\frac{1}{p^{2}} \frac{\partial}{\partial p}\left(p^{2} f_{p} E \cos \theta_{p}\right)-\frac{1}{p \sin \theta_{p}} \frac{\partial}{\partial \theta_{p}}\left(\sin ^{2}\left(\theta_{p}\right) f_{p} E\right)\right) \\
+\frac{n_{\mathrm{QD}}}{(2 \pi)^{2} \hbar} \iiint\left(f_{p^{\prime}}-f_{p}\right)\left|\tilde{V}\left(\vec{p}^{\prime}-\vec{p}\right)\right|^{2} \delta\left(\varepsilon_{p^{\prime}}-\varepsilon_{p}\right) d^{3} p^{\prime}
\end{array}\right)
\end{aligned}
$$

Under the influence of electric field, the final form of the drift velocity can be written and calculated as

$$
\langle v\rangle=\frac{\iint \frac{\hbar p \cos \theta_{p}}{m} f\left(p, \theta_{p}\right) p^{2} \sin \theta_{p} d k d \theta_{p}}{\iint f\left(p, \theta_{p}\right) p^{2} \sin \theta_{p} d p d \theta_{i}}
$$

As the electric field and electron velocity have a linear relation at low fields, therefore the slope of the linear part of the velocity-field curve is the carrier mobility.

\section{Results}

Figure 2 depicts, in each time step, the electron velocity while updating until it reaches a steady state and gets to be autonomous on time. The dependence of velocity on the electric field is determined at different values of the applied electric field, and subsequently, the carrier mobility is calculated from the slope of the velocity-electric field relation. As the figure shows, increasing the electric field increases the velocity effectively, and it takes more time steps to reach a constant carrier velocity. This calculated for a truncated conical InAs/ GaAs QDIP with QD density of 673 $\mu \mathrm{m}^{-2}$ while the radii of the bottom and top bases of the truncated conical QD are $8.132 \mathrm{~nm}$ and $3.253 \mathrm{~nm}$, respectively and the QD height is equal to $9.615 \mathrm{~nm}$. For an operating temperature of $77 \mathrm{~K}$, the electron mobility of the device will be $0.0636 \mathrm{~m}^{2} /$ (V.S). 


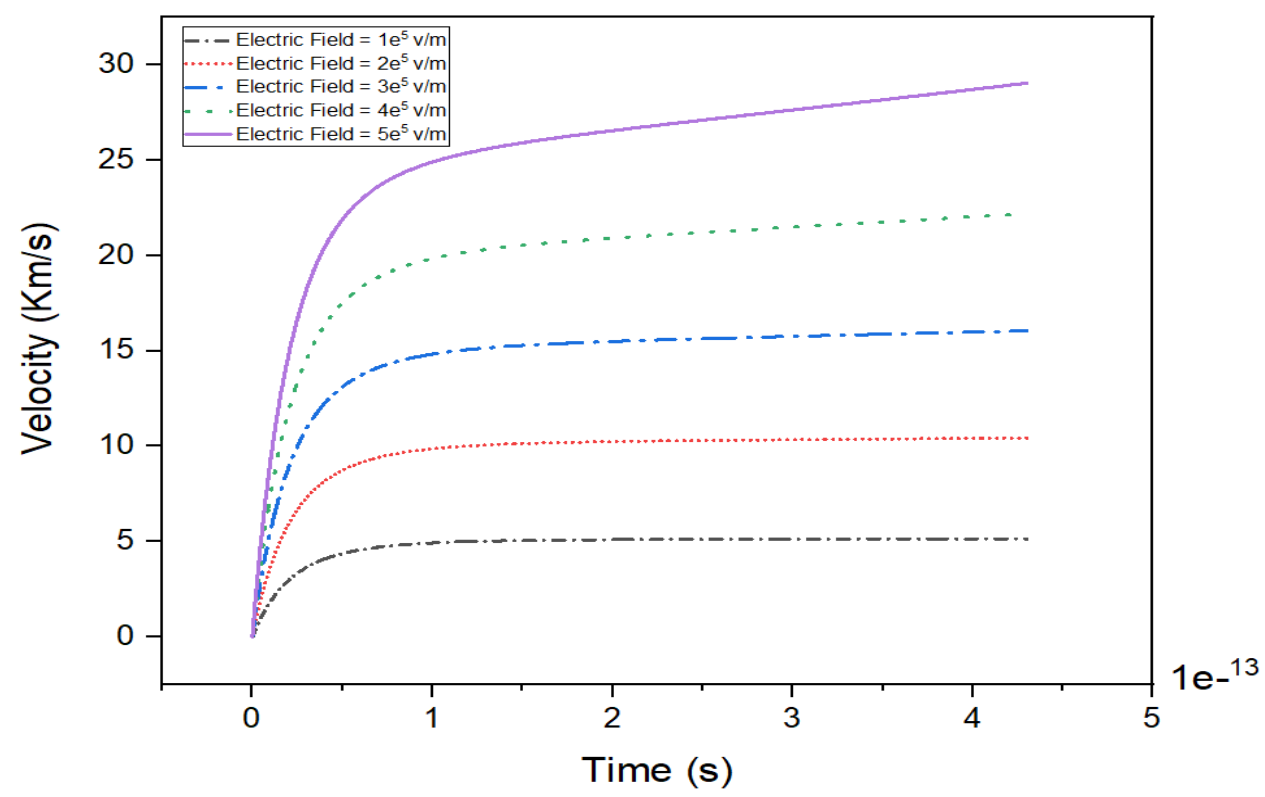

Figure 2: The electron velocity versus number of time steps for different applied electric field values

The influence of the density and dimensions of QDs on the carrier mobility are studied in both Figure 3 and Figure 4. These characteristics have been studied for QDIP described in Figure 1 (a), which comprises 5 layers of truncated conical InAs QDs, while GaAs is the barrier material, and the operating temperature of the photodetector is assumed as $77 \mathrm{~K}$. In Figure 3 depicts the mobility on the QD height $h_{d}$ for different values of the QD densities is shown where the QD base radius is kept at $r_{d}=8.13173 \mathrm{~nm}$. While Figure 4 depicts the mobility dependence on the QD base radius $r_{d}$ for different values of QD density while the QD height is kept at $h_{d}=10 \mathrm{~nm}$. As predicted, mobility declines with the increase of the radius, density, and height of the QD, as these combined factors lead to an increase of the surface area in the plane that is normal to the electric field direction, which results in slowing down the carriers due to the increase of number of the scattering events. These characteristics and the effects of the dimensions and the surface area of the truncated conical QD on the carrier mobility is shown in

\section{Table 1.}

Figure 5 shows the electron velocity versus applied electric field characteristics for both truncated conical, conical, hemispherical and spherical, QDs of identical volumes. For these different quantum dot structures, as illustrated, the slope in case of the truncated conical QDs is smaller than the slope for conical QD case but larger than the slope for hemispherical or spherical QDs. Also, by decreasing the aspect ratio of the truncated conical QD $\left(r_{o} / r_{d}\right)$, the mobility increases as it approaches the conical shape. This behavior is shown in details in Table 2, where the carrier mobility of truncated conical, conical, hemispherical, and spherical QDs with the same volume, materials, operating temperature, and density are compared. 
As the diagram shows, there is an inverse correlation between expanding the surface area and the carrier mobility, because an enlarged contact locale increases the collision probability between the QD's surface and the carrier. It is noteworthy that there is a substantial difference between the carrier mobility values for truncated conical, conical, and hemispherical QDs on the one hand and the values of the spherical QDs on the other, which emphasizes that the necessity of solving the BTE without approximations.

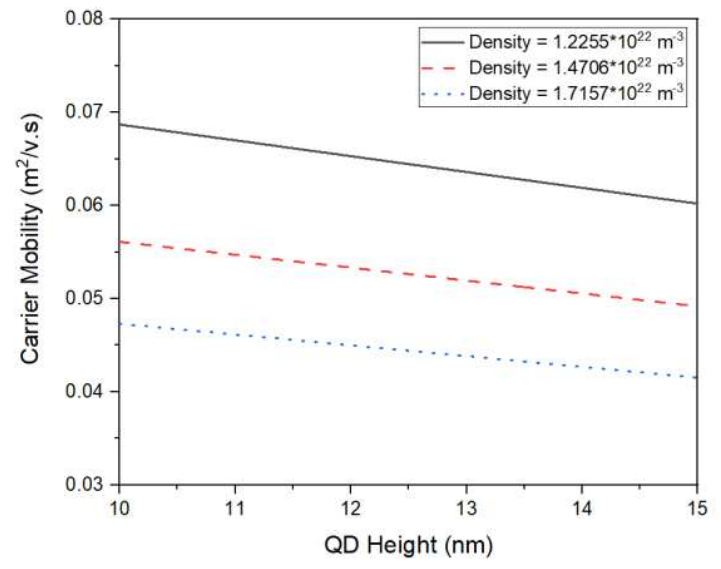

Figure 3: Carrier mobility versus the height of a truncated conical for various densities

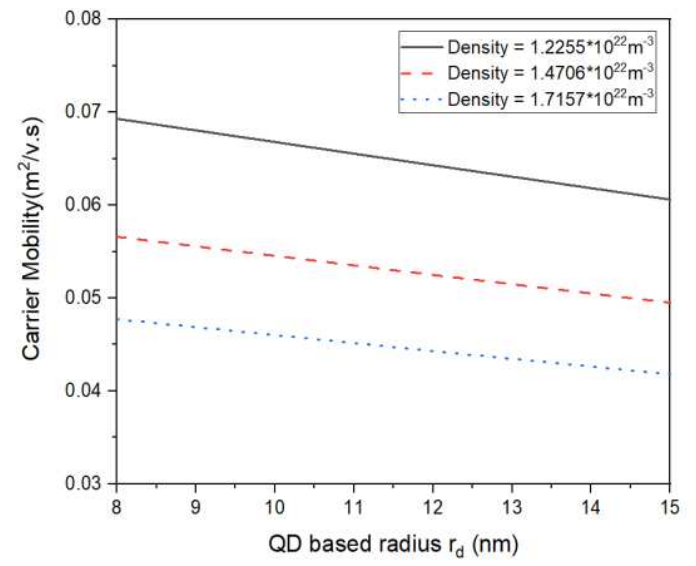

Figure 4: Carrier mobility versus radius of a truncated conical for various densities

Table 1: Truncated conical mobility of QDs with changing height under the same aspect ratio for $\mathrm{T}=77$ $\mathrm{K}$

\begin{tabular}{|c|c|c|c|c|c|c|}
\hline $\begin{array}{l}\text { Form of } \\
\text { QD }\end{array}$ & $\begin{array}{l}\text { Dimensions } \\
\text { of QD } \\
\mathrm{nm}\end{array}$ & $\begin{array}{l}\text { Volume } \\
\text { of QD } \\
\mathbf{n m}^{3}\end{array}$ & $\begin{array}{l}\text { Type of } \\
\text { Materials }\end{array}$ & $\begin{array}{c}\text { Density } \\
\text { Of QD } \\
\mu^{-2}\end{array}$ & $\begin{array}{l}\text { Mobility } \\
\mathbf{m}^{2} /(\text { V.S })\end{array}$ & $\begin{array}{l}\text { Surface } \\
\text { Area }\end{array}$ \\
\hline $\begin{array}{l}\text { Truncated } \\
\text { Conical }\end{array}$ & $\begin{array}{l}r_{d 1}=8.1317 \\
r_{d 2}=4.0658 \\
h_{d}=10\end{array}$ & 1211.8045 & $\mathrm{InAs} / \mathrm{GaAs}$ & 673 & 0.0542 & 673.3340 \\
\hline $\begin{array}{l}\text { Truncated } \\
\text { Conical }\end{array}$ & $\begin{array}{l}r_{d 1}=8.1317 \\
r_{d 2}=4.0658 \\
h_{d}=12\end{array}$ & 1454.1654 & InAs/GaAs & 673 & 0.0503 & 745.18877 \\
\hline $\begin{array}{l}\text { Truncated } \\
\text { Conical }\end{array}$ & $\begin{array}{l}r_{d 1}=8.1317 \\
r_{d 2}=4.0658 \\
h_{d}=15\end{array}$ & 1817.7067 & InAs/GaAs & 673 & 0.0474 & 855.212 \\
\hline
\end{tabular}




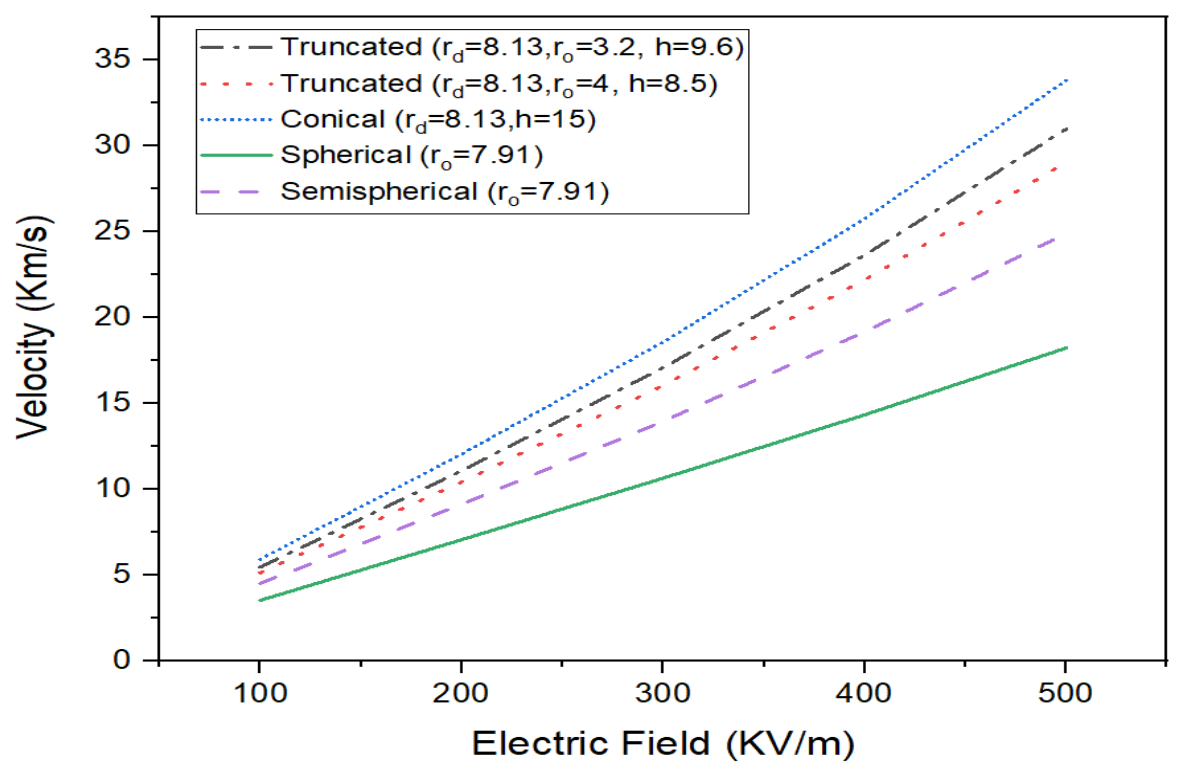

Figure 5: The carrier velocity versus applied electric field for different QD shapes

Table 2: The carrier mobility values under truncated conical, conical, spherical and hemispherical QDs of identical volume where $T=77 \mathrm{~K}$

\begin{tabular}{llclcl}
\hline $\begin{array}{l}\text { Form of } \\
\text { QD }\end{array}$ & $\begin{array}{l}\text { Dimensions } \\
\text { of QD } \\
\mathbf{n m}\end{array}$ & $\begin{array}{c}\text { Volume } \\
\mathbf{o f} \mathbf{Q D} \\
\mathbf{n m}^{\mathbf{3}}\end{array}$ & $\begin{array}{l}\text { Type of } \\
\text { Materials }\end{array}$ & $\begin{array}{c}\text { Density } \\
\mathbf{o f} \mathbf{Q D} \\
\mathbf{m m}^{-2}\end{array}$ & $\begin{array}{l}\text { Mobility } \\
\mathbf{m}^{2} /(\mathbf{V} . \mathbf{S})\end{array}$ \\
\hline Cone & $\begin{array}{l}r_{d}=8.1317 \\
h_{d}=15\end{array}$ & 1038.69 & InAs/GaAs & 673 & 0.0695 \\
\hline $\begin{array}{l}\text { Truncated } \\
\text { conical }\end{array}$ & $\begin{array}{l}r_{d 1}=8.1317 \\
r_{d 2}=3.2526 \\
h_{d}=9.615\end{array}$ & 1038.69 & InAs/GaAs & 673 & 0.0636 \\
\hline $\begin{array}{l}\text { Truncated } \\
\text { conical }\end{array}$ & $\begin{array}{l}r_{d 1}=8.1317 \\
r_{d 2}=4.0658\end{array}$ & 1038.69 & InAs/GaAs & 673 & 0.0596 \\
\hline Sphere & $h_{d}=8.571$ & & & & \\
\hline Hemisphere & $r_{o}=6.2825$ & 1038.69 & InAs/GaAs & 673 & 0.0413 \\
\hline & $r_{o}=7.9154$ & 1038.69 & InAs/GaAs & 673 & 0.0508 \\
\hline
\end{tabular}




\section{Conclusions}

In the current research, a full theoretical model is introduced to determine the carrier mobility through a truncated conical QDIP. Carrier mobility is an important factor influencing major detector's parametric functions i.e. drift velocity, electrical conductivity and dark current. The truncated conical QD is the most likely shaped to enable the self-assembled QDs. The Boltzmann transport equation was numerically solved in the presented model to obtain precise carrier mobility using the time-domain finite difference method. The interdependence of QDs dimensions and densities on the carrier mobility is examined in this work. QD size or density are inversely proportional to the carrier mobility, as the scattering events number increases leading to a slowdown of the carriers, leading to the decrease of the device's photocurrent and dark current. Additionally, the carrier mobility of the truncated conical QD is contrasted to conical, spherical, and hemispherical QD of similar QD volume, type of material, and density at the same operating temperature. Calculating the diverse structures of QD using the proposed theoretical method of mobility calculation is a considerable step to predict the behavior of QDIPs.

\section{References}

[1] P. Dardano and M. A. Ferrara, "Integrated photodetectors based on Group IV and colloidal semiconductors: Current state of affairs," Micromachines, vol. 11, no. 9, 2020, doi: 10.3390/MI11090842.

[2] V. P. Deviprasad et al., "Spatial Optimization of Modulation Doping in P-I-P QDIPs: Towards Achieving Higher Operating Temperature," IEEE Trans. Nanotechnol., vol. 19, no. c, pp. 247-254, 2020, doi: 10.1109/TNANO.2019.2937093.

[3] H. Ghimire, P. V. V. Jayaweera, D. Somvanshi, Y. Lao, and A. G. U. Perera, "Recent progress on extended wavelength and split-offband heterostructure infrared detectors," Micromachines, vol. 11, no. 6, pp. 1-16, 2020, doi: 10.3390/mi11060547.

[4] A. V. Barve, S. J. Lee, S. K. Noh, and S. Krishna, "Review of current progress in quantum dot infrared photodetectors," Laser Photonics Rev., vol. 4, no. 6, pp. 738-750, 2010, doi: 10.1002/lpor.200900031.

[5] S. Sengupta and S. Chakrabarti, Structural, optical and spectral behaviour of InAs-based quantum dot heterostructures: Applications for high-performance infrared photodetectors. 2017.

[6] P. Martyniuk and A. Rogalski, "Quantum-dot infrared photodetectors: Status and outlook," Progress in Quantum Electronics, vol. 32, no. 3-4. pp. 89-120, 2008, doi: 10.1016/j.pquantelec.2008.07.001.

[7] T. A. A. Y. M. El-batawy, "Bound to continuum absorption coefficient for spherical and conical quantum dots," pp. 149-157, 2015, doi: 10.1007/s11082-014-9894-2.

[8] H. C. Liu, M. Gao, J. McCaffrey, Z. R. Wasilewski, and S. Fafard, "Quantum dot infrared 
photodetectors," Appl. Phys. Lett., vol. 78, no. 1, pp. 79-81, 2001, doi: 10.1063/1.1337649.

[9] H. Liu, Q. Tong, G. Liu, C. Yang, and Y. Shi, "Performance characteristics of quantum dot infrared photodetectors under illumination condition," Opt. Quantum Electron., vol. 47, no. 3, pp. 721-733, 2015, doi: 10.1007/s11082-014-9947-6.

[10] H. Lim et al., "Quantum dot infrared photodetectors: Comparison of experiment and theory," Phys. Rev. B - Condens. Matter Mater. Phys., vol. 72, no. 8, pp. 1-15, 2005, doi: 10.1103/PhysRevB.72.085332.

[11] T. A. Ameen and Y. M. El-Batawy, "Polarization dependence of absorption by bound electrons in self-assembled quantum dots," J. Appl. Phys., vol. 113, no. 19, 2013, doi: 10.1063/1.4805061.

[12] T. A. Ameen, Y. M. El-Batawy, and A. A. Abouelsaood, "Modeling of the quantum dot filling and the dark current of quantum dot infrared photodetectors," J. Appl. Phys., vol. 115, no. 6, 2014, doi: 10.1063/1.4864762.

[13] M. A. Naser, M. J. Deen, and D. A. Thompson, "Photocurrent Modeling and Detectivity Optimization in a Resonant-Tunneling Quantum-Dot Infrared Photodetector," vol. 46, no. 6, pp. 849-859, 2010.

[14] K. Banoo, A. Technologies, C. Shu, and J. W. Jerome, "Simulating Quasi-ballistic Transport in Si Nanotransistors," no. August 2000, 2001, doi: 10.1155/2001/16023.

[15] Y. M. El-Batawy and A. Hosny, "Modeling of carrier mobility for semispherical quantum dot infrared photodetectors (QDIPs)," Opt. Quantum Electron., vol. 52, no. 2, Feb. 2020, doi: 10.1007/s11082-019-2170-8.

[16] S. Youssef, Y. M. El-Batawy, and A. A. Abouelsaood, "Effect of self assembled quantum dots on carrier mobility, with application to modeling the dark current in quantum dot infrared photodetectors," J. Appl. Phys., vol. 120, no. 12, 2016, doi: 10.1063/1.4963287.

[17] B. K. Ridley, Quantum Processes in Semiconductors, 4th edn. Oxford University Press Inc., New York (1999). . 


\section{Declarations}

\section{Funding}

The authors declare that they did not receive any funding

\section{Conflicts of interest/Competing interests}

The authors declare that they have no competing interests

\section{Availability of data and material}

Not applicable

\section{Code availability}

The authors declare that they have made their custom code

\section{Ethics approval}

Not applicable

\section{Consent to participate}

Not applicable

\section{Consent for publication}

Not applicable 
Figures

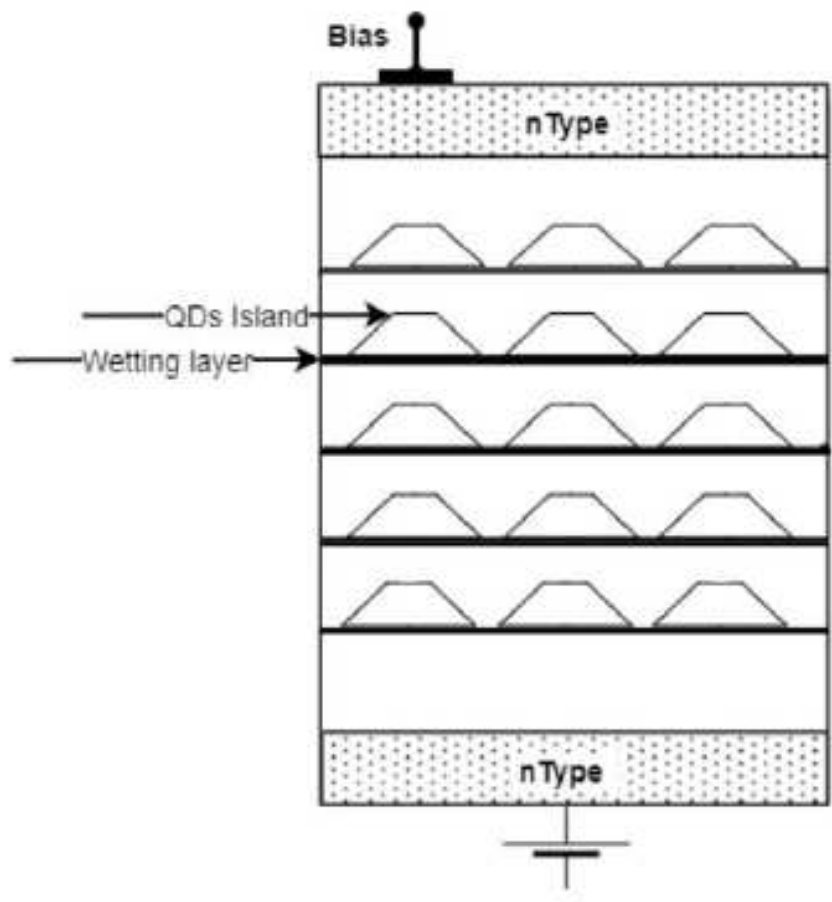

(a)

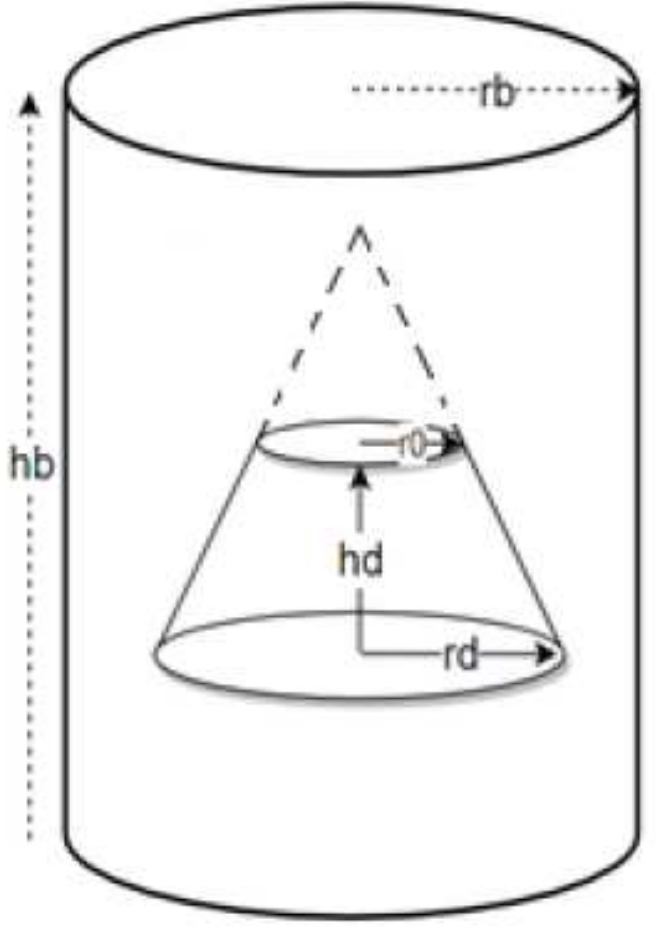

(b)

Figure 1

(a) Truncated conical QDIP structure (b) Basic cell of truncated conical QD structure 


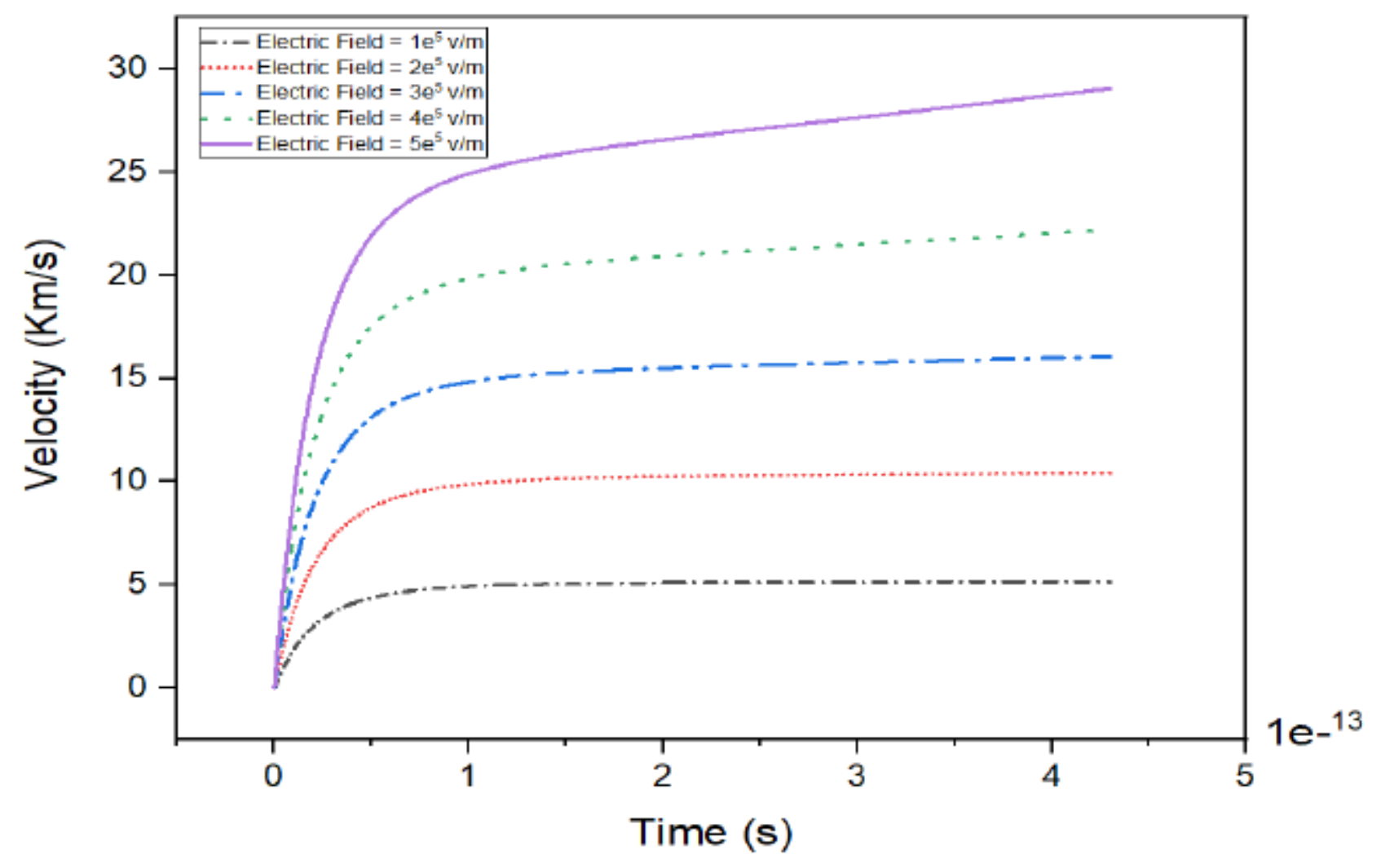

Figure 2

The electron velocity versus number of time steps for different applied electric field values

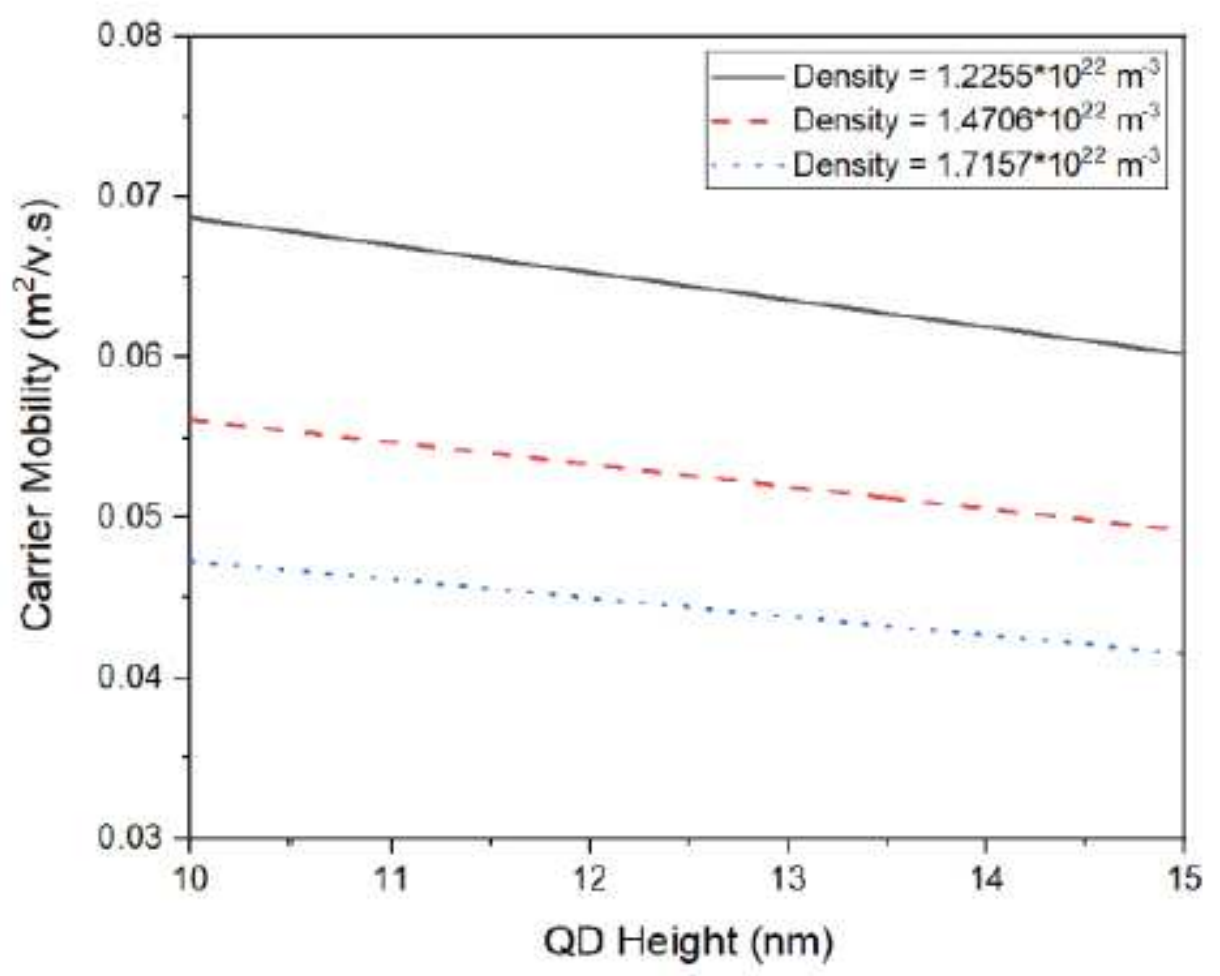

Figure 3 
Carrier mobility versus the height of a truncated conical for various densities

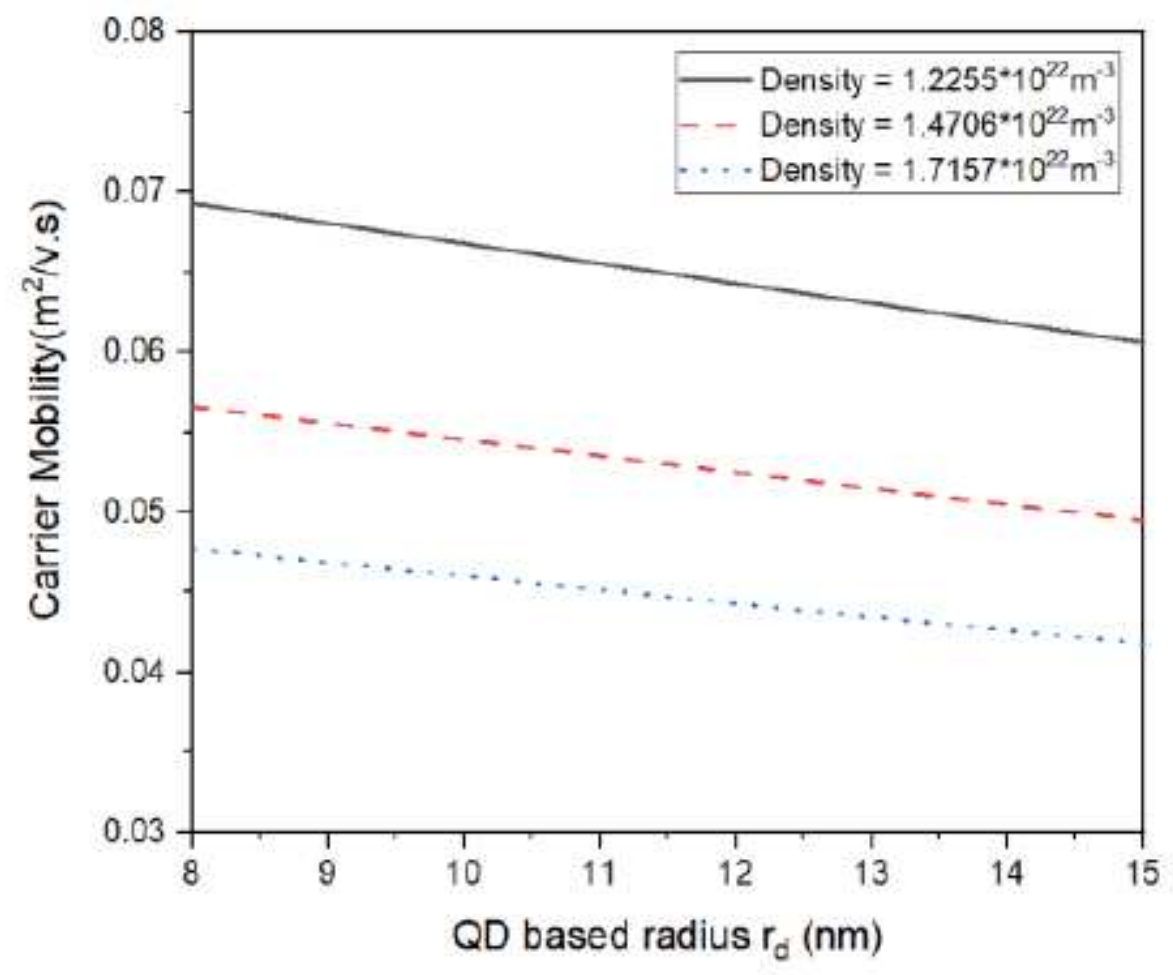

Figure 4

Carrier mobility versus radius of a truncated conical for various densities

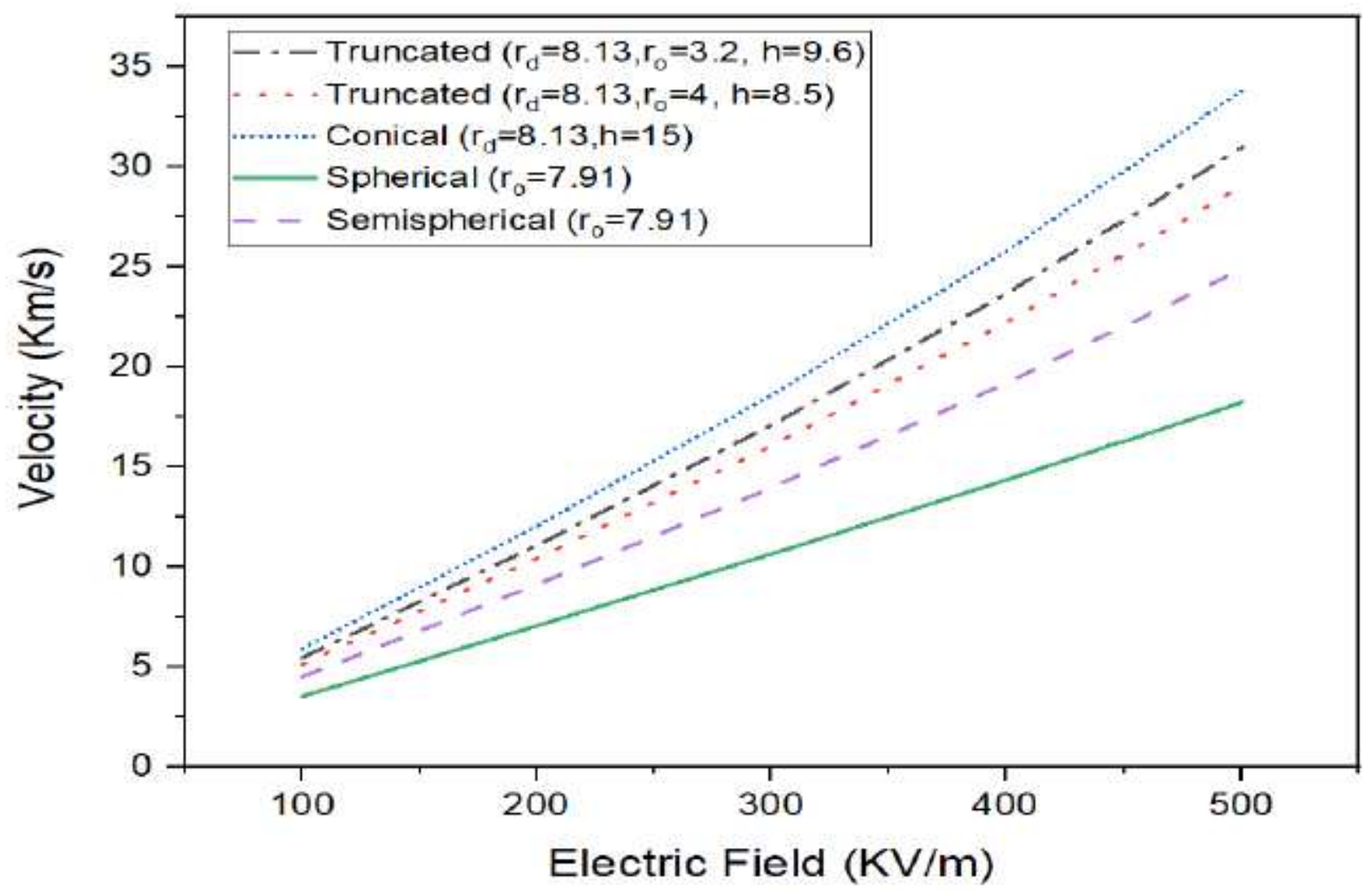


Figure 5

The carrier velocity versus applied electric field for different QD shapes 